\title{
A safety and efficacy analysis of direct-antiviral therapy in patients with chronic hepatitis $\mathbf{C}$ after renal transplantation
}

\author{
Guanghui Li, Junjie Ma, Lu Xu, Jiali Fang, Lei Zhang, Jialin Wu, Xingqiang Lai, Luhao Liu, Yunyi Xiong, \\ Wei Yin, Tao Zhang, Peng Zhang, Li Li, Rongxin Chen, Jiao Wang, Zheng Chen \\ Department of Organ Transplantation, Second Affiliated Hospital of Guangzhou Medical University, Guangzhou 510260, China \\ Contributions: (I) Conception and design: G Li; (II) Administrative support: J Ma, Z Chen; (III) Provision of study materials or patients: L Xu, J Fang, \\ L Zhang, X Lai, L Liu, Y Xiong, W Yin, T Zhang, P Zhang, L Li, R Chen; (IV) Collection and assembly of data: G Li, J Wu, J Wang; (V) Data \\ analysis and interpretation: G Li, Z Chen; (VI) Manuscript writing: All authors; (VII) Final approval of manuscript: All authors. \\ Correspondence to: Zheng Chen. Department of Organ Transplantation, Second Affiliated Hospital of Guangzhou Medical University, No. 250, \\ Changgang East Road, Haizhu District, Guangzhou 510260, China. Email: docchenzheng@163.com.
}

\begin{abstract}
Background: To explore the safety and efficacy of direct antiviral therapy in patients with hepatitis $\mathrm{C}$ virus (HCV) infection after renal transplantation.

Methods: We retrospectively reviewed the medical information of 15 cases of $\mathrm{HCV}^{+}$patients treated with direct antiviral therapy after renal transplantation in our center. The effectiveness of direct antiviral therapy was evaluated by analyzing the HCV-RNA levels of patients at 1, 4, 12, 24, and 48 weeks before and after antiviral therapy. In addition, parameters including the rejection rate, the blood concentration of anti-rejection drugs, liver function level [alanine aminotransferase (ALT), aspartate transaminase (AST)], estimated glomerular filtration rate (eGFR) and serum creatinine (CREA) levels were used to assess its safety. Results: A total of 15 patients were enrolled in the study. All patients turned HCV-RNA negative after 12 weeks of direct-antiviral therapy; the serological test of all patients demonstrated an $100 \%$ response rate in rapid virological response (RVR) (15/15), 12-week sustained virological response (SVR12), and 24-week sustained virological response (SVR24). In addition, compared to pre-treatment, the liver function within 12, 24, and 48 weeks after treatment was significantly improved. Moreover, eGFR, CREA, and anti-rejection drug concentration remained stable while acute rejection reaction and other obvious side effects were not observed throughout the treatment period.
\end{abstract}

Conclusions: The direct antiviral therapy was well-tolerated and effective for patients with chronic hepatitis $\mathrm{C}$ after renal transplantation.

Keywords: Renal transplantation; chronic hepatitis C; direct antiviral drugs; virological response

Submitted Sep 04, 2019. Accepted for publication Dec 04, 2019.

doi: $10.21037 /$ tau.2020.01.05

View this article at: http://dx.doi.org/10.21037/tau.2020.01.05

\section{Introduction}

Hepatitis $\mathrm{C}$ virus (HCV) infection is a global public health problem with an infection rate of approximately $2.5 \%$ and the number of infected people approaching 180 million (1). Blood is the main means of HCV transmission, and chronic kidney disease (CKD) patients with regular hemodialysis are prone to $\mathrm{HCV}$ infection due to the requirements of establishing extracorporeal circulation pathways for blood and the use of blood products. Studies have shown that the incidence of $\mathrm{HCV}$ infection in CKD patients is significantly higher than in normal people (2). HCV infection was also reported to be closely related to the occurrence of membrane proliferative glomerulonephritis with cryoglobulinemia (3). Therefore, The Kidney Disease Improving Global Outcomes (KDIGO) recommends that CKD patients with $\mathrm{HCV}$ infection be regularly checked for creatinine clearance and proteinuria status and receive 
antiviral therapy (4). Studies have demonstrated that renal transplantation has a longer survival than hemodialysis in patients with end-stage renal disease and HCV infection (5). However, other research has found that the long-term survival rates of human/graft were lower in kidney transplant recipients with $\mathrm{HCV}$ infection than in non-HCV infection recipients (6), which was mainly due to the poor tolerance and low sustained virological response (SVR) to pegylated interferon alpha (PEG IFN-a) and ribavirin before the advent of direct antiviral agents (DAAs). DAAs have replaced interferon as the standard treatment for chronic hepatitis $\mathrm{C}$ based on their ability to effectively eliminate $\mathrm{HCV}$ and improve or reduce the progress of the disease for the purpose of treatment (7). However, there are few clinical reports about the application of such drugs in patients with renal transplantation, while the mechanism of their interaction with conventional immunosuppressive agents such as tacrolimus and mycophenolic acid remain unclear. Furthermore, investigations into the safety and efficacy of DAAs in the management of $\mathrm{HCV}$ patients after renal transplantation in a large sample size are also lacking. Thus, we reviewed the medical records of $\mathrm{HCV}^{+}$patients who received DAAs after renal transplantation in our center from January 2016 to July 2018.

\section{Methods}

\section{Inclusion and exclusion criterium}

\section{Inclusion criteria}

Patients received renal transplantation with complete follow-up data in our center from January 2016 to December 2018; patients diagnosed with HCV infection before renal transplantation under diagnostic criteria based on the "Hepatitis $C$ prevention guidelines (updated in 2015)" issued by the Chinese Medical Association Liver Diseases Society (8); no prior treatment with DAA; baseline estimated glomerular filtration rate (eGFR) levels before antiviral therapy higher than $30 \mathrm{~mL} / \mathrm{min} / 1.73 \mathrm{~m}^{2}$ ); no contraindications for taking DAA.

\section{Exclusion criteria}

Patients co-infected with human immunodeficiency virus (HIV); patients with HCV combined with other viral hepatitis; patients with incomplete medical records.

\section{Treatment regimen of $\mathrm{HCV}$}

Since PEG IFN-a was closely related to post-transplant rejection, all patients in our center had complete $\mathrm{HCV}$ genotyping (HCV-GT) before kidney transplantation, and DAA treatment (excluding PEG IFN-a) was started after stabilizing renal function. The Asian Pacific Association for the Study of the Liver (APASL) Guidelines recommend that different HCV-GT treatments require different DAAs (9). Thus, the specific protocols were as follows: HCV-GT1a patients used a combination of sofosbuvir $400 \mathrm{mg} / \mathrm{d}$ + ledipasvir $90 \mathrm{mg} / \mathrm{d}$; HCV-GT-1b patients received a compound dosage form of sofosbuvir $400 \mathrm{mg} / \mathrm{d}+$ ledipasvir $90 \mathrm{mg} / \mathrm{d}$ or sofosbuvir $400 \mathrm{mg} / \mathrm{d}$ + daclatasvir $60 \mathrm{mg} / \mathrm{d}$; HCVGT-3b patients were treated with sofosbuvir $400 \mathrm{mg} / \mathrm{d}+$ daclatasvir $60 \mathrm{mg} / \mathrm{d}$; HCV-GT-6a patients were treated with sofosbuvir $400 \mathrm{mg} / \mathrm{d}+$ ledipasvir $90 \mathrm{mg} / \mathrm{d}$. Patients began DAA treatment with a 12 -week cycle after their condition stabilized which was considered to be the taking of a maintenance dose of immunosuppressant, stable graft function, and no complications such as infection.

\section{Assessment of the general clinical status}

According to the post-operation interval, a comprehensive follow-up was performed every 1-4 weeks, including physical examination, medication, liver and kidney function, blood routine, drug concentration, HCV-RNA, and eGFR calculation using the Chronic Kidney Disease Epidemiology Collaboration (CKD-EPI) formula (10).

\section{Virological assessment}

If the recipients presented with positive $\mathrm{HCV}$ antibody when entering the waiting list, serum HCV-RNA detection was required, and the serum HCV-RNA test was performed again before the kidney transplantation to confirm the diagnosis. The serum HCV-RNA was detected at 1, 4, 12, 24, and 48 weeks after DAA treatment, and the serum HCV antibody was detected after 12 weeks of DAA treatment. The virological response indicators after anti-chronic HCV therapy are shown in Table 1.

\section{Statistical analysis}

SPSS 17.0 was used for statistical analysis. Continuous variables conformed to a normal distribution are expressed as mean \pm standard deviation $(\bar{x}+\mathrm{s})$; Student's unpaired $t$-test was used to compare continuous variables, and categorical variables were compared using chi-square test $\left(\chi^{2}\right.$-test). $\mathrm{P}<0.05$ was considered as statistically significant. 
Table 1 Definition of virological response indicators for antiviral treatment of chronic hepatitis C virus (HCV)

\begin{tabular}{ll}
\hline Terms & Definition \\
\hline RVR & HCV RNA below the LLD at week 4 of treatment \\
EVR & HCV RNA decreased by >2 Log at week 12 compared with pre-treatment \\
cEVR & HCV RNA below the LLD at week 12 of treatment \\
pEVR & HCV RNA decreased by >2 Log but higher than LLD at week 12 of treatment compared with pre-treatment \\
DVR & Based on pEVR, HCV RNA lower than LLD by week 24 of treatment \\
PR & Based on pEVR, HCV RNA still higher than LLD by week 24 of treatment \\
NR & HCV RNA decreased by <2 Log at week 12 compared with pre-treatment \\
Breakthrough & HCV RNA rebound during treatment after achieving virological response \\
Relapse & After achieving a virological response, HCV-RNA can be detected within 24 weeks of treatment \\
SVR & HCV-RNA was lower than LLD within 12 and 24 weeks after treatment, and was recorded as SVR12 and SVR24 \\
\hline respectively.
\end{tabular}

RVR, rapid virological response; LLD, lower limit of normal detection; EVR, early virological response; cEVR, complete early virological response; pEVR, partial early virological response; DVR, delayed virological response; PR, partial response; NR, no response; SVR, sustained virological response.

\section{Results}

\section{Baseline characteristics (detailed in Table 2)}

A total of 15 patients were enrolled in the study, all of whom were diagnosed with chronic end-stage renal disease and $\mathrm{HCV}^{+}$and received $\mathrm{HCV}^{-}$donor kidneys for transplantation. There were 11 males and 4 females. The age at time of transplantation ranged between 33 and $65(48.8 \pm 13.6)$ years old. Before surgery, 14 patients presented with positive anti-HCV antibodies while 1 patient presented negative; the HCV-RNA load was greater than $10^{3}$; all patients were to receive their first kidney transplant; no clinical symptoms of cirrhosis was observed in patients with or without abnormal liver function indexes before surgery.

\section{The use of immunosuppressive drugs}

The immunosuppressive regimens of 15 patients with renal transplantation were as follows: D0 methylprednisolone $500 \mathrm{mg}+$ Thymoglobuline $50 \mathrm{mg}$, D1 methylprednisolone $500 \mathrm{mg}+$ Thymoglobuline $50 \mathrm{mg}$, D2 methylprednisolone $250 \mathrm{mg}+$ Thymoglobuline $25 \mathrm{mg}$, D3 methylprednisolone $250 \mathrm{mg}+$ Thymoglobuline $25 \mathrm{mg}$. The immunosuppressive regimens after renal transplantation were as follows: 15 patients received tacrolimus + mycophenolate mofetil + methylprednisolone. The initial dose of tacrolimus was $0.6-0.8 \mathrm{mg} / \mathrm{kg} / \mathrm{d}$, and the tacrolimus dose was adjusted according to the blood concentration. The tacrolimus concentration was maintained at $5-10 \mathrm{ng} / \mathrm{mL}$ within 3 months after surgery; The concentration of tacrolimus was maintained at $5-10 \mathrm{ng} / \mathrm{mL}$ after 3 months, the initial dose of mycophenolate mofetil was $1.0-1.5 \mathrm{~g} / \mathrm{d}$, and the maintenance dose was $1.0 \mathrm{~g} / \mathrm{d}$. Methylprednisolone was initiated at a dose of $20 \mathrm{mg} / \mathrm{d}$, and reduced by $4 \mathrm{mg}$ every 3 days and finally maintained at $8 \mathrm{mg} / \mathrm{d}$.

\section{Efficacy}

All patients received DAA treatment; HCV-RNA levels decreased to the normal range at about 1 week (detailed in Figure 1). Sustained virologic response (SVR) and HCV-RNA levels lower than the minimum limit of detection (LLD) at 12 and 24 weeks after treatment were considered as indexes for efficacy evaluation. DAAs were selected for different HCV-GT treatments based on the recommendations of the Asian-Pacific Association for the Study of the Liver (APASL) guidelines (detailed in Table 3). The SVR12 and SVR24 levels after antiviral therapy are listed in Table 4.

\section{Safety and tolerance}

No adverse events associated with direct antiviral drugs were observed throughout the study period (detailed in Tables 5-7). 
Table 2 Baseline characteristics of enrolled patients

\begin{tabular}{lc}
\hline Variables & General information \\
\hline Sex & Male: 11 cases; female: 4 cases \\
Age at transplantation (years) & $33-65(48.8 \pm 13.6)$ \\
HCV antibody & Positive: 14 cases; negative: 1 case \\
HCV-RNA & Higher than $10^{3}$ \\
HCV genotypes & $1 \mathrm{a}: 2$ cases; $1 \mathrm{~b}: 8$ cases; $3 \mathrm{~b}: 3$ cases; $6 \mathrm{a}: 2$ cases \\
\hline
\end{tabular}

HCV, hepatitis C virus.

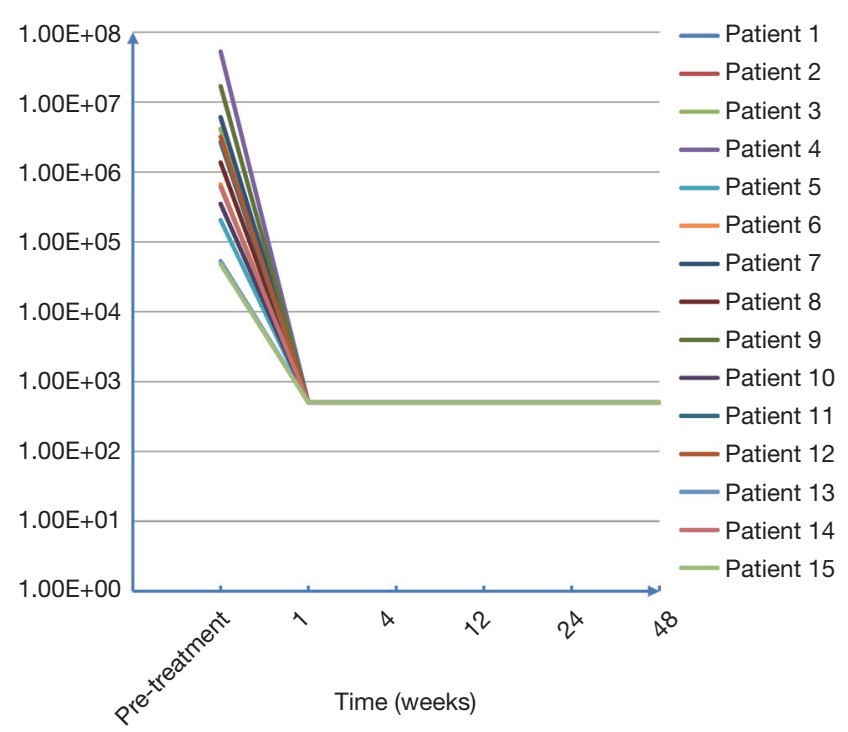

Figure $1 \mathrm{HCV}-\mathrm{RNA}$ in 15 patients before and after DDA treatment. All patients received DAA treatment; HCV-RNA levels decreased to the normal range at about 1 week. $\mathrm{HCV}$, hepatitis $\mathrm{C}$ virus; DAA, direct antiviral agent.

\section{Discussion}

$\mathrm{HCV}$ is a flavivirus of the genus hepatitis, the genome of which is a single-stranded positive RNA characterized by significant heterogeneity and high variability (11). The analysis of HCV strains of all known genomic sequences demonstrate that the nucleotide and amino acid sequences are quite different among HCV strains and the degree of variation in each part of the genome is also inconsistent (11). The high variability of $\mathrm{HCV}$ has made it difficult to develop a vaccine which can effectively prevent hepatitis $\mathrm{C}$, and patients infected with a certain type of HCV have poor immunity, allowing them to be reinfected by HCVs of different genotypes. Currently, $\mathrm{HCV}$ can be divided into at least 6 genotypes and multiple subtypes. In accordance with internationally accepted methods, $\mathrm{HCV}$ genotypes are represented by Arabic numerals, and gene subtypes are expressed in lower case letters (12). The HCV genotypes of Chinese patients have been reported to be mainly type $1 \mathrm{~b}$ (56.8\%), while type 2 accounts for $24.1 \%$, type 3 accounts $9.1 \%$, type 6 accounts for just $6.3 \%$, and types 4 and 5 are absent in the population. HCV-GT follow-up in our center revealed 2 cases of type 1a (2/15, accounting for $13.3 \%)$, 8 cases of type $1 \mathrm{~b}(8 / 15$, accounting for $53.3 \%), 3$ cases of type $3 \mathrm{~b}(3 / 15$, accounting for $20.0 \%)$, and 2 cases of type $6 \mathrm{a}$ (2/15, accounting for $13.3 \%)(13)$.

At present, HCV infection is more common in hemodialysis and infusion of blood products, and there are also some donor-derived infections among transplant patients (14). Chronic hepatitis $\mathrm{C}$ after renal transplantation is a significant cause of liver disease and a high-risk factor for cirrhosis, liver cancer, and death after transplantation; it is also an independent risk factor affecting long-term kidney-related survival post-transplantation (15). In addition, HCV infection has also been associated with proteinuria, renal allograft, hepatitis $\mathrm{C}$-associated nephritis, and chronic rejection after renal transplantation. Thus, it is of great importance to treat these patients with antiviral therapy (16).

PEG IFN-a still remains in the main scheme of anti-viral treatment of hepatitis $\mathrm{C}$ according to the $\mathrm{HCV}$ prevention and treatment guidelines in China (2015 edition). However, its application in $\mathrm{HCV}^{+}$patients after renal transplant has been limited to a large extent by the risk it poses in inducing rejection of solid transplanted organs and poor tolerance in kidney transplant patients. Although DAAs have achieved significant efficacy in a non-general population (17), clinical data of DAAs in the treatment of patients with kidney transplantation and $\mathrm{HCV}^{+}$are still rare. The most concerning questions for transplant doctors 
Table 3 Usage of direct antiviral drugs for different hepatitis $\mathrm{C}$ viruses

\begin{tabular}{lclc}
\hline Type of virus & Number of cases & Drug regiment & Clinical efficacy \\
\hline $1 a$ & 2 & Sofosbuvir + ledipasvir & RVR + SVR \\
$1 b$ & 5 & Sofosbuvir + ledipasvir & RVR + SVR \\
$1 b$ & 3 & Sofosbuvir + daclatasvir & RVR + SVR \\
$3 b$ & 3 & Sofosbuvir + daclatasvir & RVR + SVR \\
$6 a$ & 2 & Sofosbuvir + ledipasvir & RVR + SVR \\
\hline
\end{tabular}

RVR, rapid virological response; SVR, sustained virological response.

Table 4 HCV antibody status before and after treatment with DAAs

\begin{tabular}{lccccc}
\hline Status & Pre-treatment & 4 weeks & 12 weeks & 24 weeks & 14 weeks \\
\hline Positive & 14 & 14 & 14 & 14 \\
Negative & 1 & 1 & 1 & 1 & 1 \\
\hline
\end{tabular}

The status of HCV antibody at each time point after treatment was consistent with that of pretreatment. HCV, hepatitis C virus; DAA, direct antiviral agent.

Table 5 Liver function (ALT, AST)

\begin{tabular}{|c|c|c|c|c|c|}
\hline Indicators & Pre-treatment & 4 weeks & 12 weeks & 24 weeks & 48 weeks \\
\hline AST (U/L) & $62 \pm 30$ & $28 \pm 9$ & $22 \pm 6$ & $22 \pm 6$ & $20 \pm 6$ \\
\hline$P$ value & - & $\mathrm{P}^{1}<0.05$ & $P^{2}>0.05$ & $P^{3}>0.05$ & $P^{4}>0.05$ \\
\hline
\end{tabular}

After DAA treatment, there were statistical differences in ALT and AST in all patients compared with those before treatment, and there were no statistical differences at different time points after treatment. $\mathrm{P}^{1}<0.05$ (statistical difference between 4 weeks of treatment and pre-treatment); $\mathrm{P}^{2}>0.05$ (no statistical difference between 12 weeks of treatment and 4 weeks); $\mathrm{P}^{3}>0.05$ (no statistical difference between 24 weeks of treatment and 4 weeks); $P^{4}>0.05$ (no statistical difference between 48 weeks of treatment and 4 weeks). ALT, alanine aminotransferase; AST, aspartate transaminase; DAA, direct antiviral agent.

Table 6 Graft function and status (CREA, eGFR, FK506-TL, and rejection)

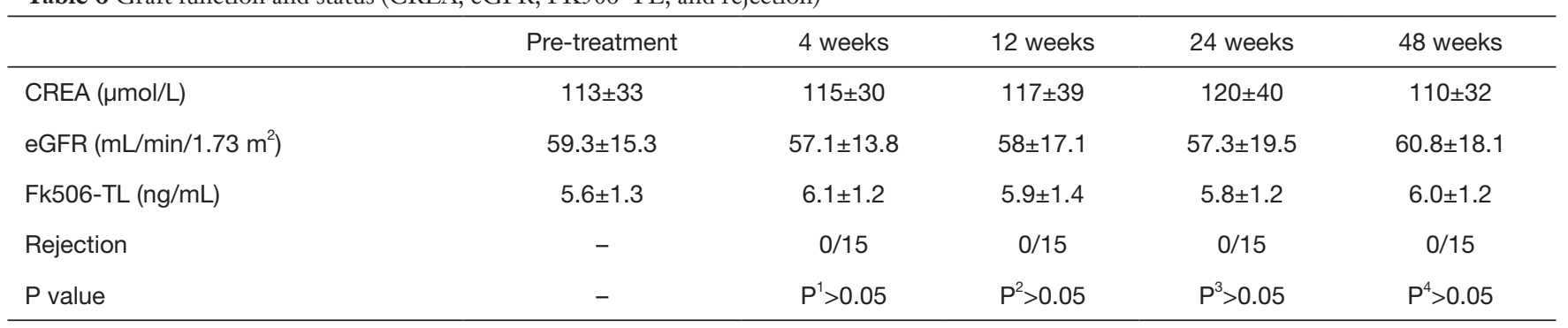

There were no statistical differences in CREA, eGFR, Fk506-TL, and rejection between receiving DAA treatment and pre-treatment. $\mathrm{P}^{1}>0.05$ (no statistical difference between 4 weeks of treatment and pre-treatment); $\mathrm{P}^{2}>0.05$ (no statistical difference between 12 weeks of treatment and pre-treatment); $\mathrm{P}^{3}>0.05$ (no statistical difference between 24 weeks of treatment and pre-treatment); $P^{4}>0.05$ (no statistical difference between 48 weeks of treatment and pre-treatment). CREA, creatinine; eGFR, estimated glomerular filtration rate; DAA, direct antiviral agent. 
Table 7 Blood routine (WBC, PLT)

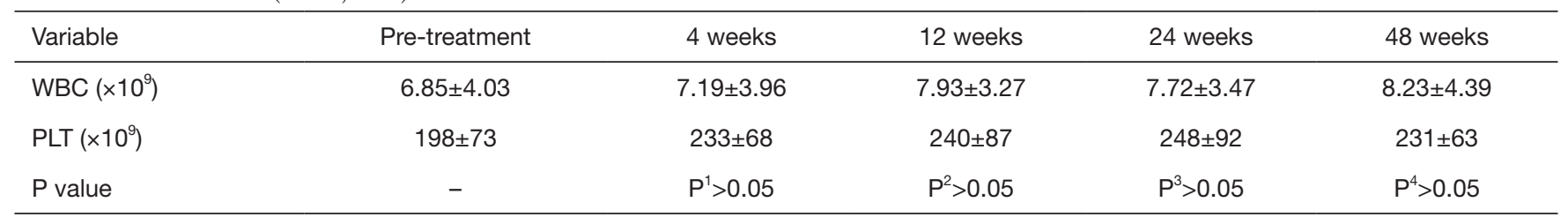

After DAA treatment, there was no statistical difference in WBC and PLT between all patients compared with those before treatment. $P^{1}>0.05$ (no statistical difference between 4 weeks of treatment and pre-treatment); $P^{2}>0.05$ (no statistical difference between 12 weeks of treatment and pre-treatment); $\mathrm{P}^{3}>0.05$ (no statistical difference between 24 weeks of treatment and pre-treatment); $\mathrm{P}^{4}>0.05$ (no statistical difference between 48 weeks of treatment and pre-treatment). WBC, white blood cell; PLT, platelet; DAA, direct antiviral agent.

are the following: (I) whether DAA requires adjustment of dose in patients with impaired liver and kidney function; (II) the nature of the interaction between DAAs and immunosuppressants; (III) the level of DAA tolerance in patients after transplantation, and whether DDAs cause other adverse events such as rejection.

In this study, all patients underwent DAA treatment after graft function recovered and condition stabilized. The median level of serum CREA was $113 \pm 33 \mu \mathrm{mol} / \mathrm{L}$, and the median level of eGFR was $59.3 \pm 15.3 \mathrm{~mL} / \mathrm{min} / 1.73 \mathrm{~m}^{2}$. DAA drug selection was based on different genotypes. Previous studies suggest sofosbuvir not be adjusted in patients with eGFR $>30 \mathrm{~mL} / \mathrm{min}$, but for patients with eGFR $<30 \mathrm{~mL} / \mathrm{min}$ or infected via hemodialysis, the recommended dose of sofosbuvir has yet to be determined (18). There is no need to adjust the daclatasvir dose in patients with renal impairment (19), nor is there a need to do so with the ledipasvir dose in patients with mild or moderate renal impairment. However, for patients with severe renal impairment and end-stage renal disease requiring hemodialysis, there has been no assessment of the safety and efficacy of ledipasvir performed thus far. A multicenter study demonstrated that antiviral therapy in patients with chronic hepatitis $\mathrm{C}$ infection were safe and effective before and after renal transplantation, and the antiviral treatment of hepatitis $\mathrm{C}$ after transplantation increased the availability of the transplant and reduced the waiting time. Furthermore, delayed treatment until post-transplantation seemed to have no adverse effect on the overall survival of the allogeneic kidney and the recipient (20). However, due to the limited amount of data reported, we believe that the optimal treatment time for patients with chronic hepatitis $\mathrm{C}$ and end-stage renal disease is when the kidney transplant is completed and renal function has recovered. The selected DAA regimen was sofosbuvir + daclatasvir and sofosbuvir/ ledipasvir (complex dosage form). All patients received conventional doses of antiviral therapy.

Since the regiment of DAA for patients with chronic hepatitis $C$ and end-stage renal disease after renal transplantation has been determined, questions have then emerged concerning whether the metabolism of DAA drugs would affect the metabolism of immunosuppressants and further affect the function of renal graft. In our study, the concentration of immunosuppressant in patients treated with DAA was not changed before and after treatment, and no interaction was found between DAA drugs and the metabolism and blood concentration of immunosuppressants. Furthermore, other studies also suggested no interaction between sofosbuvir and immunosuppressants including tacrolimus, mycophenolate mofetil, prednisone, cyclosporine or azathioprine (21). In addition to sofosbuvir and ribavirin, sofosbuvir/ledipasvir has also been approved for the treatment of hepatitis $\mathrm{C}$ patients after liver transplantation, and no interaction with these immunosuppressive agents has been found. Our results also showed that the grafts status, including serum CREA, eGFR and other indicators presented no significant statistical differences before and after treatment. No graft rejection was found during the clinical observation period in all patients who received DAA treatment. Therefore, it was safe to treat patients with $\mathrm{HCV}+$ end-stage renal disease with DAAs after receiving a kidney transplant.

Although investigations about the effectiveness of DAA drug treatment after organ transplantation are rare, all previous studies concerning the therapeutic effect of DAA in renal transplant patients were in accordance with our expectations, and our results were similar to previous studies. Both SVR12 and SVR24 were $100 \%$, and all patients had a continuous viral response after 48 weeks of follow-up. In addition, the follow-up results also demonstrated that the transaminase before treatment and the liver function index post-treatment were significantly 
improved. All the above results initially indicate that the existing DAA regimens can achieve significant efficiency in $\mathrm{HCV}^{+}$patients after kidney transplantation. However, due to the small sample size of current studies, a multicenter prospective study in a larger scale is still required to further confirm the validity. Mao et al. reported that seven patients with renal transplantation and $\mathrm{HCV}^{+}$were treated with sofosbuvir $400 \mathrm{mg} / \mathrm{d}$ + daclatasvir $60 \mathrm{mg} / \mathrm{d}$, all of whom achieved satisfactory clinical outcomes including an $100 \%$ SVR24 level and no adverse effects of DAAs on renal function were observed (22).

Since some DAAs have not yet been listed in China, the clinical research in this field has been severely restricted. Considering the differences across ethnic populations and the HCV genotype distribution across continents, reports from non-Chinese contexts were not fully applicable to our research. Our study provides a reference for the DAAs used in China and suggests that the DAA regiment based on sofosbuvir is relatively stable and safe in the management of chronic hepatitis $\mathrm{C}$ infection in patients with endstage renal disease after renal transplantation. We believe that DAAs will provide new opportunities and choices for patients with chronic hepatitis $\mathrm{C}$ by virtue of their limited effects and significant clinical efficacy. However, a multicenter large-scale prospective study is still required in the near future to further validate these conclusions.

\section{Acknowledgments}

Funding: None.

\section{Footnote}

Conflicts of Interest: All authors have completed the ICMJE uniform disclosure form (available at http://dx.doi. org/10.21037/tau.2020.01.05). The authors have no conflicts of interest to declare.

Ethical Statement: The authors are accountable for all aspects of the work in ensuring that questions related to the accuracy or integrity of any part of the work are appropriately investigated and resolved. The study was approved by the Ethics Committee of the Second Affiliated Hospital of Guangzhou Medical University and the number of the approval was 2015025. All patients had signed informed consent forms before participation in this study.

Open Access Statement: This is an Open Access article distributed in accordance with the Creative Commons Attribution-NonCommercial-NoDerivs 4.0 International License (CC BY-NC-ND 4.0), which permits the noncommercial replication and distribution of the article with the strict proviso that no changes or edits are made and the original work is properly cited (including links to both the formal publication through the relevant DOI and the license). See: https://creativecommons.org/licenses/by-nc$\mathrm{nd} / 4.0 /$.

\section{References}

1. Solli P, Dolci G, Ranieri VM. The new frontier of hepatitis $\mathrm{C}$ virus (HCV)-mismatched heart and lung transplantation. Ann Transl Med 2019;7:S279.

2. Cacoub P, Gragnani L, Comarnond C, et al. Extrahepatic manifestations of chronic hepatitis $\mathrm{C}$ virus infection. Dig Liver Dis 2014;46:S165-73.

3. Shah HH, Patel C. Long-term response to peginterferon in hepatitis $\mathrm{C}$ virus-associated nephrotic syndrome from focal segmental glomerulosclerosis. Ren Fail 2013;35:1182-5.

4. Kidney Disease: Improving Global Outcomes (KDIGO). KDIGO clinical practice guidelines for the prevention, diagnosis, evaluation, and treatment of hepatitis C in chronic kidney disease. Kidney Int Suppl 2008;(109):S1-99.

5. Ingsathit A, Kamanamool N, Thakkinstian A, et al. Survival advantage of kidney transplantation over dialysis in patients with hepatitis $\mathrm{C}$ : a systematic review and metaanalysis. Transplantation 2013;95:943-48.

6. Maluf DG, Fisher RA, King AL, et al. Hepatitis C virus infection and kidney transplantation: Predictors of patient and graft survival. Transplantation 2007;83:853-57.

7. Jakobsen JC, Nielsen EE, Feinberg J, et al. Direct-acting antivirals for chronic hepatitis C. Cochrane Database Syst Rev 2017;6:CD012143.

8. Chinese Society of Hepatology, Chinese Medical Association, Wei L, et al. The guideline of prevention and treatment for hepatitis C: a 2015 update. Zhonghua Gan Zang Bing Za Zhi 2015;23:906-23.

9. Omata M, Kanda T, Wei L, et al. APASL consensus statements and recommendation on treatment of hepatitis C. Hepatol Int 2016;10:702-26.

10. Levey AS, Stevens LA, Schmid CH, et al. A new equation to estimate glomerular filtration rate. Ann Intern Med 2009;150:604-12.

11. Zeuzem S, Foster GR, Wang S, et al. Glecaprevir- 
Pibrentasvir for 8 or 12 Weeks in HCV Genotype 1 or 3 Infection. N Engl J Med 2018;378:354-69.

12. Simmonds P, Bukh J, Combet C, et al. Consensus proposals for a unified system of nomenclature of hepatitis C virus genotypes. Hepatology 2005;42:962-73.

13. Ju W, Yang S, Feng S, et al. Hepatitis C virus genotype and subtype distribution in Chinese chronic hepatitis $\mathrm{C}$ patients: nationwide spread of HCV genotypes 3 and 6. Virol J 2015;12:109.

14. Morales JM, Fabrizi F. Hepatitis $\mathrm{C}$ and its impact on renal transplantation. Nat Rev Nephrol 2015;11:172-82.

15. Boratyńska $M$, Wakulenko A, Klinger $M$, et al. Chronic allograft dysfunction in kidney transplant recipients: longterm single-center study. Transplant Proc 2014;46:2673-7.

16. Domínguez-Gil B, Morales JM. Transplantation in the patient with hepatitis C. Transpl Int 2009;22:1117-31.

17. Marshall AD, Pawlotsky JM, Lazarus JV, et al. The removal of DAA restrictions in Europe - One step closer to eliminating $\mathrm{HCV}$ as a major public health threat. J

Cite this article as: $\mathrm{Li} \mathrm{G}, \mathrm{Ma} \mathrm{J}$, Xu L, Fang J, Zhang L, Wu J, Lai X, Liu L, Xiong Y, Yin W, Zhang T, Zhang P, Li L, Chen R, Wang J, Chen Z. A safety and efficacy analysis of directantiviral therapy in patients with chronic hepatitis $\mathrm{C}$ after renal transplantation. Transl Androl Urol 2020;9(2):583-590. doi: 10.21037/tau.2020.01.05
Hepatol 2018;69:1188-96.

18. Charlton M, Gane E, Manns MP, et al. Sofosbuvir and ribavirin for treatment of compensated recurrent hepatitis $\mathrm{C}$ virus infection after liver transplantation. Gastroenterology 2015;148:108-17.

19. Cornpropst MT, Denning JM, Clemons D, et al. The effect of renal impairment and end stage renal disease on the single-dose pharmacokinetics of PSI-7977. J Hepatol 2012;56:S433.

20. Chascsa DM, Mousa OY, Pungpapong S, et al. Clinical outcomes of hepatitis $\mathrm{C}$ treatment before and after kidney transplantation and its impact on time to transplant: A multicenter study. Am J Transplant 2018;18:2559-65.

21. Poole RM. Daclatasvir + asunaprevir: first global approval. Drugs 2014;74:1559-71.

22. Mao Y, Zhang X, Liu S, et al. Efficacy and safety of direct-acting antiviral therapy for hepatitis $\mathrm{C}$ after renal transplantation. Journal of Shandong University (Health Sciences) 2019;57:75-80. 